\title{
A study of the correlation of insulin resistance and leptin with inflammatory factors and vascular endothelial injury in T2DM patients with CHD
}

\author{
JIE ZHANG $^{1^{*}}$, JING JIN $^{2 *}$, JILUN LIU ${ }^{3}$, YAJUN HE $^{3}$, PENG ZHANG $^{4}$, \\ WUCHENG $\mathrm{YE}^{5}$, WEI ZHU ${ }^{6}$ and MINGLIANG $\mathrm{LI}^{7}$ \\ Departments of ${ }^{1}$ Cardiology and ${ }^{2}$ Geriatrics, Xi'an No. 5 Hospital, Xi'an, Shaanxi 710082; \\ ${ }^{3}$ Department of Cardiology, No. 215 Hospital of Shaanxi Nuclear Industry, Xianyang, Shaanxi 712000; \\ ${ }^{4}$ Department of Cardiology, The Affiliated Hospital of Yan'an University, Yan'an, Shaanxi 716000; \\ ${ }^{5}$ Department of Cardiology, Xingyuan Hospital of Yulin, Yulin, Shaanxi 719000; \\ ${ }^{6}$ Department of Clinical Laboratory, The Central Hospital of Baoji, Baoji, Shaanxi 721008; \\ ${ }^{7}$ Department of Cardiovascular Medicine, Hanzhong People's Hospital, \\ Hanzhong, Shaanxi 723000, P.R. China
}

Received August 28, 2017; Accepted April 17, 2018

DOI: $10.3892 / \mathrm{etm} .2018 .6170$

\begin{abstract}
The purpose of this study was to explore the correlation of insulin resistance (IR) and leptin with inflammatory factors and vascular endothelial injury in patients with type 2 diabetes mellitus (T2DM) complicated with coronary heart disease (CHD) was explored. One hundred and fifty normal patients (normal group), 150 patients with pure T2DM (T2DM group) and 150 patients with T2DM complicated with coronary heart disease (T2DM + CHD group) were selected from Xi'an No. 5 Hospital. All the participants met our inclusion criteria. Age, body mass index, waist-to-hip ratio, blood lipid and fasting plasma glucose (FPG), of all the subjects were measured. Chemiluminescent immunoassay was adopted for the detection of FPG and double-antibody sandwich method was used for the determination of fasting plasma leptin, and assay of high-sensitivity $\mathrm{C}$-reactive protein (hs-CRP), interleukin-6 (IL-6) and tumor necrosis factor- $\alpha$ $(\mathrm{TNF}-\alpha)$. Insulin resistance index (IRI) was used to evaluate IR and enzyme-linked immunosorbent assay was adopted for the detection of von Willebrand factor (vWF) and endothelin (ET-1). Compared with the control group, patients in the
\end{abstract}

Correspondence to: $\mathrm{Dr}$ Mingliang Li, Department of Cardiovascular Medicine, Hanzhong People's Hospital, 251 Bei Tuanjie Street, Hanzhong, Shaanxi 723000, P.R. China

E-mail: limingliang082@163.com

*Contributed equally

Key words: type 2 diabetes mellitus complicated with coronary heart disease, insulin resistance, leptin, inflammatory factors, vascular endothelial injury
T2DM + CHD group and those in the T2DM group had higher homeostasis model assessment-IR, and higher assay of plasma leptin, hs-CRP, IL-6 and TNF- $\alpha(\mathrm{P}<0.05)$, and lower vascular endothelial function $(\mathrm{P}<0.05)$. Moreover, compared with the T2DM group, T2DM + CHD group had higher plasma leptin, and higher assay of hs-CRP, IL- 6 and TNF- $\alpha(\mathrm{P}<0.05)$. IRI was positively correlated with hs-CRP ( $\mathrm{r}=0.521, \mathrm{P}=0.001)$, IL-6 $(r=0.359, P=0.001)$ and TNF- $\alpha(r=0.386, P=0.001)$, leptin was positively correlated with hs-CRP $(\mathrm{r}=0.305, \mathrm{P}=0.001)$, IL-6 ( $r=0.259, P=0.002)$ and TNF- $\alpha(r=0.429, P=0.001)$, and IRI had no correlation with ET-1 $(r=0.058, \mathrm{P}=0.734)$ and vWF $(r=0.047, P=0.812)$, that is, it had no direct correlation with vascular endothelial function. Level of leptin was positively correlated with ET-1 $(\mathrm{r}=0.366, \mathrm{P}=0.001)$ and $\mathrm{vWF}$ $(\mathrm{r}=0.471, \mathrm{P}=0.001)$, that is, it was negatively correlated with vascular endothelial function. Our results showed that leptin, hs-CRP, IL- 6 and TNF- $\alpha$ are involved in the occurrence and development of CHD in patients with T2DM. IR has no direct correlation with the occurrence and development of CHD in patients with T2DM.

\section{Introduction}

Type 2 diabetes mellitus (T2DM) is a metabolic disease that occurs frequently mainly in the middle-aged and elderly period and accounts for above $90 \%$ of diabetes. Diabetes often leads to cardiovascular disease (1). The morbidity and mortality rates of T2DM complicated with coronary heart disease (CHD) are increasing. Approximately $70 \%$ of T2DM eventually die of cardiovascular and cerebrovascular diseases, and CHD is responsible for $50 \%$ of deaths (2). Occurrence and development mechanism of CHD in patients with T2DM is not clear yet. Increasing number of studies are conducted on insulin resistance (IR), oxidative stress, disorder of lipid metabolism, endothelial dysfunction, hypertension and 
Table I. Comparison of general data and test results of biochemical indexes among the groups (mean $\pm \mathrm{SD}$ ).

\begin{tabular}{|c|c|c|c|}
\hline \multirow[b]{2}{*}{ Item } & \multicolumn{3}{|c|}{ Groups } \\
\hline & Normal & T2DM & $\mathrm{T} 2 \mathrm{DM}+\mathrm{CHD}$ \\
\hline No. & 150 & 150 & 150 \\
\hline Age (years) & $50.40 \pm 8.56$ & $51.69 \pm 7.78$ & $52.40 \pm 8.42$ \\
\hline \multicolumn{4}{|l|}{$\operatorname{Sex}(n)$} \\
\hline Male & 75 & 76 & 74 \\
\hline Female & 75 & 74 & 76 \\
\hline BMI $\left(\mathrm{kg} / \mathrm{m}^{2}\right)$ & $23.42 \pm 3.56$ & $23.78 \pm 3.24$ & $28.12 \pm 3.13^{\mathrm{a}, \mathrm{b}}$ \\
\hline FPG (mmol/l) & $5.04 \pm 0.62$ & $9.35 \pm 1.24^{\mathrm{a}}$ & $13.32 \pm 1.62^{\mathrm{a}, \mathrm{b}}$ \\
\hline WHR & $0.82 \pm 0.04$ & $0.96 \pm 0.04^{\mathrm{a}}$ & $0.97 \pm 0.06^{\mathrm{a}}$ \\
\hline $\mathrm{SBP}(\mathrm{mm} \mathrm{Hg})$ & $124.80 \pm 14.36$ & $130.96 \pm 12.56$ & $161.42 \pm 17.56^{\mathrm{a}, \mathrm{t}}$ \\
\hline DBP (mm Hg) & $80.14 \pm 9.08$ & $87.54 \pm 9.13^{\mathrm{a}}$ & $94.43 \pm 10.13^{\mathrm{a}, \mathrm{b}}$ \\
\hline FINS (mU/l) & $5.76 \pm 0.63$ & $11.28 \pm 2.05^{\mathrm{a}}$ & $15.72 \pm 2.64^{\mathrm{a}}$ \\
\hline HbA1c (\%) & $5.31 \pm 0.33$ & $8.29 \pm 0.51^{\mathrm{a}}$ & $10.88 \pm 2.01^{\mathrm{a}, \mathrm{b}}$ \\
\hline \multirow[t]{2}{*}{ HOMA-IR } & 1.38 & 2.79 & 3.52 \\
\hline & $(0.52-1.84)$ & $(0.84-3.41)^{\mathrm{a}}$ & $(1.72-4.86)^{\mathrm{a}, \mathrm{b}}$ \\
\hline $\begin{array}{l}\text { Triglyceride } \\
(\mathrm{mmol} / \mathrm{l})\end{array}$ & $1.45 \pm 0.42$ & $1.61 \pm 0.46$ & $1.68 \pm 0.56$ \\
\hline $\begin{array}{l}\text { Total cholesterol } \\
(\mathrm{mmol} / \mathrm{l})\end{array}$ & $4.88 \pm 0.87$ & $4.90 \pm 1.08$ & $4.74 \pm 1.10$ \\
\hline $\mathrm{HDL}-\mathrm{C}(\mathrm{mmol} / \mathrm{l})$ & $1.43 \pm 0.27$ & $1.31 \pm 0.21$ & $0.73 \pm 0.18^{\mathrm{a}, \mathrm{b}}$ \\
\hline LDL-C (mmol/l) & $2.83 \pm 0.79$ & $3.72 \pm 0.88^{\mathrm{a}}$ & $3.69 \pm 0.93^{\mathrm{a}}$ \\
\hline Lipoprotein (a) & $4.38 \pm 0.52$ & $4.52 \pm 1.21$ & $5.43 \pm 0.96^{\mathrm{a}, \mathrm{b}}$ \\
\hline \multicolumn{4}{|l|}{$(\mathrm{mg} / \mathrm{l})$} \\
\hline hs-CRP (mg/l) & $5.72 \pm 2.22$ & $5.89 \pm 3.43$ & $16.28 \pm 19.62^{\mathrm{a}, \mathrm{b}}$ \\
\hline Leptin $(\mu \mathrm{g} / \mathrm{l})$ & $1.86 \pm 0.34$ & $2.08 \pm 0.54^{\mathrm{a}}$ & $2.42 \pm 0.62^{\mathrm{a}, \mathrm{b}}$ \\
\hline ET-1 (mmol/l) & $35 \pm 9$ & $49 \pm 13^{a}$ & $61 \pm 19^{\mathrm{a}, \mathrm{b}}$ \\
\hline TNF- $\alpha(\mathrm{ng} / \mathrm{ml})$ & $0.25 \pm 0.12$ & $0.41 \pm 0.26^{\mathrm{a}}$ & $0.73 \pm 0.38^{\mathrm{a}, \mathrm{b}}$ \\
\hline IL-6 (ng/ml) & $0.33 \pm 0.28$ & $0.73 \pm 0.24^{\mathrm{a}}$ & $1.08 \pm 0.32^{\mathrm{a}, \mathrm{b}}$ \\
\hline $\mathrm{vWF}(\%)$ & $112.65 \pm 20.62$ & $130.54 \pm 22.64^{\mathrm{a}}$ & $150.68 \pm 24.76^{\mathrm{a}, \mathrm{t}}$ \\
\hline
\end{tabular}

${ }^{\mathrm{a}} \mathrm{P}<0.05$, compared with normal group; ${ }^{\mathrm{b}} \mathrm{P}<0.05$, compared with $\mathrm{T} 2 \mathrm{DM}$ group. T2DM, type 2 diabetes mellitus; T2DM+CHD, type 2 diabetes mellitus complicated with coronary heart disease; BMI, body mass index; FPG, fasting plasma glucose; WHR, waist-to-hip ratio; SBP, systolic blood pressure; DBP, diastolic blood pressure; FINS, fasting insulin; HbA1c, hemoglobin A1c; HDL-C, high-density lipoprotein cholesterol; LDL-C, low-density lipoprotein cholesterol; hs-CRP, high-sensitivity C-reactive protein; ET-1, endothelin; TNF- $\alpha$, tumor necrosis factor- $\alpha$; IL-6, interleukin-6; HOMA-IR, homeostasis model assessment-insulin resistance; vWF, von Willebrand factor.

inflammatory reaction $(3,4)$. It was shown that IR is a common pathophysiological change of metabolic syndrome, diabetes and atherosclerosis. When IR occurs, the concentration of inflammatory cytokines and inflammatory-sensitive proteins in the body will increase, leading to the inflammatory reaction in the arterial wall (5). This study mainly explored the correlation of IR and leptin with inflammatory factors and vascular endothelial injury in patients with T2DM + CHD with the expectation of providing a novel diagnostic indicator for of diabetes complicated with CHD.

\section{Patients and methods}

General data. One hundred and fifty normal patients, 150 patients with T2DM and 150 patients with T2DM + CHD were selected from Xi'an No. 5 Hospital (Xi'an, China). General data of the three groups of patients are given in Table I.

Inclusion and exclusion criteria .

Inclusion criteria: Patients with T2DM: Diagnostic criteria for diabetes established by WHO in 1999 was taken as the reference of diagnosis; patients with T2DM + CHD: Patients that had diabetes mellitus complicated with CHD and the diagnostic criteria established by International Heart Association and WHO in 1979 were taken for the determination of CHD; normal group: Patients that were free from diabetes mellitus and CHD.

Exclusion criteria: i) Patients with type 1 diabetes mellitus or diabetes mellitus with complications; patients with resistant or primary hypertension or patients with hypertension with left ventricle hypertrophy; patients with peripheral vascular diseases; patients who were taking glucose-reducing, lipid-decreasing and antihypertensive drugs or using insulin for treatment. ii) Patients with hyperglycemia caused by other reasons or patients with acute or chronic inflammatory disease; patients with severe chronic complications such as heart failure and left ventricular ejection fraction $<50 \%$; patients who suffered from cerebrovascular accident. iii) Patients with endocrine diseases such as hyperthyroidism and gout; pregnant women; patients with autoimmune diseases; patients with family histories of infectious diseases and hereditary diseases. All the patients or their families signed the informed consent form and this study was approved by the Ethics Committee of Xi'an No. 5 Hospital.

Study methods. The venous blood specimens were taken from the patients on an empty stomach in the morning. Sex, age, fasting plasma glucose (FPG), waist-to-hip ratio (WHR), body mass index (BMI), blood lipid, systolic blood pressure (SBP) and diastolic blood pressure (DBP), of all the subjects were measured or collected, in which, BMI = body mass $(\mathrm{kg}) /$ height $\left(\mathrm{m}^{2}\right)$. Plasma glucose was measured by glucose oxidase method with Roche glucometer; blood lipid was measured by enzymatic method. The reagent was provided by Zhejiang Dongou Biochemical Co., Ltd., (Zhejiang, China) and the instrument used was 7170S fully-automatic biochemical analyzer provided by Hitachi, Ltd. (Tokyo, Japan); hemoglobin Alc (HbAlc) was measured by high-performance liquid chromatography (the kit was provided by Bio-Rad Laboratories, Inc., Hercules, CA, USA); fasting insulin (FINS) was measured by chemoluminescence immunoassay (the kit was provided by China Institute of Atomic Energy, Beijing, China and the instrument used was Abbotti 1000SR fully-automatic chemiluminescence analyzer provided by Abbott Pharmaceutical Co. Ltd., Lake Bluff, IL, USA); plasma leptin levels were determined by double-antibody sandwich method (plasma leptin kits were provided by Shenzhen Yinuojin Biotechnology Co., Ltd., and microplate reader was provided by Bio-Rad Laboratories, Inc.); high-sensitivity C-reactive protein (hs-CRP) was detected by turbidimetry (the kit was provided by DADE Corporation and the instrument 
used was BN ProSpec, a special Pr analyzer); von Willebrand factor (vWF), interleukin-6 (IL-6) and tumor necrosis factor- $\alpha$ (TNF- $\alpha$ ) were detected by double-antibody sandwich enzyme-linked immunosorbent assay. Endothelin (ET-1) kits were purchased from Nanjing Jiancheng Bioengineering Institute (Nanjing, China) and other kits were provided by Beijing United Bowei Biotechnology Co., Ltd., (Beijing, China). Homeostasis model assessment-insulin resistance $($ HOMA-IR $)=$ FINS/22.5 (6) and insulin resistance index $(\mathrm{IRI})=\mathrm{FPG}(\mathrm{mmol} / \mathrm{l}) \times \mathrm{FINS}(\mathrm{mU} / \mathrm{l}) / 22.5^{[2]}$ were used to evaluate IR.

Statistical analysis. All the data were processed using Statistical Product and Service Solutions (SPSS) version 22.0 (IBM Corp., Armonk, NY, USA) and the data that were in line with normal distribution are expressed as mean \pm SD. The t-test was conducted for comparison between groups and the non-normally-distributed measurement data were expressed as median (interquartile range). Non-parametric rank sum test was conducted for the comparison between groups. The $\chi^{2}$ test was adopted for comparison of rate, and variance analysis was used for comparison of the mean among multiple groups. One-way analysis of variance was used for comparison between two groups and the post-hoc test used was Tukey test. $\mathrm{P}<0.05$ was considered to indicate a statistically significant difference. Pearson's correlation test was used for the analysis on the correlation of IR and leptin with inflammatory factors and vascular endothelial injury.

\section{Results}

Comparisons of general data and relevant biochemical indexes among the three groups. General data such as constituent ratio of age and sex had no statistical significance $(P>0.05)$, and they were comparable. The differences of the comparison between the T2DM group (patients with T2DM) and the normal group in terms of biochemical indexes including FPG, DBP, FINS, HbA1c, HOMA-IR, low-density lipoprotein cholesterol (LDL-C), ET-1, TNF- $\alpha$, IL-6 and vWF had statistical significance $(\mathrm{P}<0.05)$. The differences of the comparison between the T2DM and the normal groups and that between the T2DM+CHD and the T2DM groups in terms of BMI, FPG, SBP, DBP, HbA1c, HOMA-IR, high-density lipoprotein cholesterol (HDL-C), hs-CRP, leptin, ET-1, TNF- $\alpha$, IL-6 and vWF had statistical significance $(\mathrm{P}<0.05)$. The difference of the comparison of WHR and FINS between the group of T2DM + CHD and the normal groups and that between the T2DM and the normal groups had statistical significance $(\mathrm{P}<0.05)$, while the difference of the comparison between the T2DM+CHD and the T2DM groups had no statistical significance $(\mathrm{P}>0.05)$. The differences of the comparison among the groups in terms of the remaining biochemical indexes had no statistical significance $(\mathrm{P}>0.05)$. Specific details are given in Table I.

Analysis on correlation of IR and leptin with inflammatory factors and vascular endothelial function. Pearson correlation analysis of IRI with IL- 6 , TNF- $\alpha$, hs-CRP and vascular endothelial function: IRI was positively correlated with IL-6,
Table II. Correlation of IRI with hs-CRP, IL-6, TNF- $\alpha$, ET-1 and vWF.

\begin{tabular}{lccccc}
\hline Variable & hs-CRP & IL-6 & TNF- $\alpha$ & ET-1 & vWF \\
\hline r & 0.521 & 0.359 & 0.386 & 0.058 & 0.047 \\
P-value & 0.001 & 0.001 & 0.001 & 0.734 & 0.812 \\
\hline
\end{tabular}

IRI, insulin resistance index; hs-CRP, high-sensitivity C-reactive protein; IL-6, interleukin-6; TNF- $\alpha$, tumor necrosis factor- $\alpha$; ET-1, endothelin; vWF, von Willebrand factor.

Table III. Correlation of leptin with hs-CRP, IL-6, TNF- $\alpha$, ET-1 and vWF.

\begin{tabular}{lccccc}
\hline Variable & hs-CRP & IL-6 & TNF- $\alpha$ & ET-1 & vWF \\
\hline r & 0.305 & 0.259 & 0.429 & 0.366 & 0.471 \\
P-value & 0.001 & 0.002 & 0.001 & 0.001 & 0.001
\end{tabular}

hs-CRP, high-sensitivity C-reactive protein; IL-6, interleukin-6; TNF- $\alpha$, tumor necrosis factor- $\alpha$; ET-1, endothelin; vWF, von Willebrand factor.

TNF- $\alpha$ and hs-CRP, and it had no direct correlation with vascular endothelial function (Table II).

Pearson correlation analysis of leptin with hs-CRP, IL-6, TNF- $\alpha$ and vascular endothelial function: Leptin revealed a positive correlation with hs-CRP, IL- 6 and TNF- $\alpha$, but was negatively correlated with vascular endothelial function (Table III).

\section{Discussion}

T2DM is a relatively common chronic inflammatory and metabolic disease, which can impair vascular endothelial function (7) through glucose toxicity, lipotoxicity and inflammatory mediators. Increasing number of patients with T2DM are complicated with CHD. This study explored the correlation of IR and leptin with inflammatory factors and vascular endothelial function.

Participants in this study were selected strictly as per the inclusion and exclusion criteria, which ensured the quality of the study. The sample size was relatively large, which increased credibility and persuasion of the results of this study. The study was approved by the Ethics Committees of the Xi'an No. 5 Hospital.

The mechanism of concurrence of CHD for T2DM is not clearyet. Lee et al believed that there is leptin resistance in T2DM patients complicated with CHD (8); el-Mesallamy et al (9), Pfützner and Forst (10) and Barzilay et al (11) stated that there is inflammatory reaction in T2DM patients complicated with CHD; Torimoto et al (12) and Zaghloul et al (13) considered that vascular endothelial function is impaired for T2DM patients complicated with CHD. IR is an important mechanism for the pathogenesis of T2DM and it is closely related with the occurrence and development of CHD. Leptin mainly has 
the function of regulating carbohydrate and lipid metabolism, insulin secretion and the synthesis of fat cells, thus reducing the weight (4). Resistance of insulin and leptin help to promote the secretion of leptin (8). Leptin receptors were also found in coronary endothelial cells (14). hs-CRP can inhibit the combination of leptin with its receptors, thereby inhibiting the effect of leptin (15). Resistance and insufficient secretion of insulin promote islet cells to secret TNF- $\alpha$ and IL-6, thus aggravating IR (8). vWF is a glycoprotein that is secreted by endothelial cells, which can mediate the adhesion of platelets on the vascular injury sites. Our study indicated that the difference of the comparison of HOMA-IR, hs-CRP, leptin, ET-1, TNF- $\alpha$, IL-6 and vWF between the T2DM and the normal groups and that between the group of T2DM + CHD and the T2DM groups had statistical significance, which was consistent with previous study results $(16,17)$. The T2DM group and the group of T2DM + CHD: HOMA-IR was positively correlated with hs-CRP, IL- 6 and TNF- $\alpha$, and it had no direct correlation with vascular endothelial function, which was consistent with other study results $(8,18,19)$; leptin was positively correlated with IL-6, TNF- $\alpha$ and hs-CRP, and it was negatively correlated with vascular endothelial function. The correlation of leptin with hs-CRP may be related with the function of leptin in activating protein kinase C (18), which is dose-dependent (20). Leptin has no obvious correlation with hs-CRP for normal persons. Only high concentration of leptin has such an effect. Leptin may cause vascular inflammatory response, thus participating in the occurrence and development of T2DM + CHD. Kosztáczky et al (21) and Maya-Monteiro et al (22) found in their studies that the higher the concentration of plasma leptin is, the more severe the degree of coronary artery disease will be. This study explored the correlations between IR, leptin and inflammatory factors and vascular endothelial injury in patients with T2DM + CHD. Previous reports only examined the relationship between some of these factors in patients with type 2 diabetes and CHD. Such as the relationship between leptin resistance and vascular endothelial function, leptin resistance leads to increased plasma leptin levels, which in turn cause endothelial dysfunction $(23,24)$. Although our study examined a number of indicators in patients with T2DM combined with with CHD and conducted correlation analyses, correlations between these indicators in T2DM patients without CHD were not discussed. Therefore, the specific mechanism of type 2 diabetes combined with CHD, and these indicators in type 2 diabetes without CHD still needed to be further studied.

In conclusion, TNF- $\alpha$, hs-CRP, IL- 6 and leptin are involved in the occurrence and development of CHD in patients with T2DM. IR may have no direct correlation with the occurrence and development of CHD in patients with T2DM. The detection of inflammatory factors such as hs-CRP, IL- 6 and TNF- $\alpha$ is of great clinical significance in evaluating the occurrence and development of CHD in patients with diabetes mellitus.

\section{Acknowledgements}

Not applicable.

\section{Funding}

No funding was received.

\section{Availability of data and materials}

The datasets used and/or analyzed during the current study are available from the corresponding author on reasonable request.

\section{Authors' contributions}

JZ, JJ, JL and ML designed the study and analyzed sex, age, fasting plasma glucose, waist-to-hip ratio, body mass index, blood lipid, systolic blood pressure and diastolic blood pressure of patients. JZ, YH, PZ, WY and WZ detected and interpreted von Willebrand factor, interleukin-6, tumor necrosis factor- $\alpha$ and insulin resistance index. All authors read and approved the final manuscript.

\section{Ethics approval and consent to participate}

All the patients or their families signed the informed consent form and this study was approved by the Ethics Committee of Xi'an No. 5 Hospital (Xi'an, China).

\section{Consent for publication}

Not applicable.

\section{Competing interests}

The authors declare that they have no competing interests.

\section{References}

1. Morel O, Kessler L, Ohlmann P and Bareiss P: Diabetes and the platelet: Toward new therapeutic paradigms for diabetic atherothrombosis. Atherosclerosis 212: 367-376, 2010.

2. Gaede P, Lund-Andersen H, Parving HH and Pedersen O: Effect of a multifactorial intervention on mortality in type 2 diabetes. N Engl J Med 358: 580-591, 2008.

3. Santi D, Giannetta E, Isidori AM, Vitale C, Aversa A and Simoni M: Therapy of endocrine disease. Effects of chronic use of phosphodiesterase inhibitors on endothelial markers in type 2 diabetes mellitus: A meta-analysis. Eur J Endocrinol 172: R103-R114, 2015.

4. Chazova TE, Masenko VP, Zykov KA and Golitsyna TIu: The role of inflammation factors in development of acute coronary syndrome in patients with type 2 diabetes mellitus and impaired glucose tolerance. Ter Arkh 79: 60-64, 2007 (In Russian).

5. Mahmoud F and Al-Ozairi E: Inflammatory cytokines and the risk of cardiovascular complications in type 2 diabetes. Dis Markers 35: 235-241, 2013.

6. Rothenbacher D, Brenner H, März W and Koenig W: Adiponectin, risk of coronary heart disease and correlations with cardiovascular risk markers. Eur Heart J 26: 1640-1646, 2005.

7. Kim JA, Montagnani M, Koh KK and Quon MJ: Reciprocal relationships between insulin resistance and endothelial dysfunction: Molecular and pathophysiological mechanisms. Circulation 113: 1888-1904, 2006.

8. Lee MJ, Yang RZ, Gong DW and Fried SK: Feeding and insulin increase leptin translation. Importance of the leptin mRNA untranslated regions. J Biol Chem 282: 72-80, 2007.

9. el-Mesallamy H, Suwailem S and Hamdy N: Evaluation of C-reactive protein, endothelin-1, adhesion molecule(s), and lipids as inflammatory markers in type 2 diabetes mellitus patients. Mediators Inflamm 2007: 73635, 2007.

10. Pfützner A and Forst T: High-sensitivity C-reactive protein as cardiovascular risk marker in patients with diabetes mellitus. Diabetes Technol Ther 8: 28-36, 2006.

11. Barzilay JI, Abraham L, Heckbert SR, Cushman M, Kuller LH, Resnick HE and Tracy RP: The relation of markers of inflammation to the development of glucose disorders in the elderly: The Cardiovascular Health Study. Diabetes 50: 2384-2389, 2001. 
12. Torimoto K, Okada Y, Mori H, Otsuka T, Kawaguchi M, Matsuda M, Kuno F, Sugai K, Sonoda S, Hajime M, et al: Effects of exenatide on postprandial vascular endothelial dysfunction in type 2 diabetes mellitus. Cardiovasc Diabetol 14: 25, 2015.

13. Zaghloul A, Al-Bukhari TA, Al-Pakistani HA, Shalaby M, Halawani SH, Bajuaifer N and Teama SH: Soluble endothelial protein $\mathrm{C}$ receptor and high sensitivity $\mathrm{C}$ reactive protein levels as markers of endothelial dysfunction in patients with type 1 and type 2 diabetes mellitus: Their role in the prediction of vascular complications. Diabetes Res Clin Pract 106: 597-604, 2014.

14. Knudson JD, Dincer UD, Zhang C, Swafford AN Jr, Koshida R, Picchi A, Focardi M, Dick GM and Tune JD: Leptin receptors are expressed in coronary arteries, and hyperleptinemia causes significant coronary endothelial dysfunction. Am J Physiol Heart Circ Physiol 289: H48-H56, 2005.

15. Chen K, Li F, Li J, Cai H, Strom S, Bisello A, Kelley DE, Friedman-Einat M, Skibinski GA, McCrory MA, et al: Induction of leptin resistance through direct interaction of C-reactive protein with leptin. Nat Med 12: 425-432, 2006.

16. Johnson JL, Fritsche-Danielson R, Behrendt M, WestinEriksson A, Wennbo H, Herslof M, Elebring M, George SJ, McPheat WL and Jackson CL: Effect of broad-spectrum matrix metalloproteinase inhibition on atherosclerotic plaque stability. Cardiovasc Res 71: 586-595, 2006.

17. Vozarova B, Weyer C, Hanson K, Tataranni PA, Bogardus C and Pratley RE: Circulating interleukin-6 in relation to adiposity, insulin action, and insulin secretion. Obes Res 9: 414-417, 2001.

18. De Rosa S, Cirillo P, Pacileo M, Di Palma V, Paglia A and Chiariello M: Leptin stimulated C-reactive protein production by human coronary artery endothelial cells. J Vasc Res 46: 609-617, 2009.
19. Ragab A, Abousamra NK, Higazy A and Saleh O: Relationship between insulin resistance and some coagulation and fibrinolytic parameters in patients with metabolic syndrome. Lab Hematol 14: 1-6, 2008.

20. Singh P, Hoffmann M, Wolk R, Shamsuzzaman AS and Somers VK: Leptin induces C-reactive protein expression in vascular endothelial cells. Arterioscler Thromb Vasc Biol 27: e302-e307, 2007.

21. Kosztáczky B, Fóris G, Paragh G Jr, Seres I, Zsiros E, Koncsos P, Balogh $Z$ and Paragh G: Leptin stimulates endogenous cholesterol synthesis in human monocytes: New role of an old player in atherosclerotic plaque formation. Leptin-induced increase in cholesterol synthesis. Int J Biochem Cell Biol 39: 1637-1645, 2007.

22. Maya-Monteiro CM, Almeida PE, D'Avila H, Martins AS, Rezende AP, Castro-Faria-Neto H and Bozza PT: Leptin induces macrophage lipid body formation by a phosphatidylinositol 3-kinase- and mammalian target of rapamycin-dependent mechanism. J Biol Chem 283: 2203-2210, 2008.

23. Korda M, Kubant R, Patton S and Malinski T: Leptin-induced endothelial dysfunction in obesity. Am J Physiol Heart Circ Physiol 295: H1514-H1521, 2008.

24. Taleb S, Herbin O, Ait-Oufella H, Verreth W, Gourdy P, Barateau V, Merval R, Esposito B, Clément K, Holvoet P, et al: Defective leptin/leptin receptor signaling improves regulatory $\mathrm{T}$ cell immune response and protects mice from atherosclerosis. Arterioscler Thromb Vasc Biol 27: 2691-2698, 2007. 